STRUCTURAL SCIENCE CRYSTAL ENGINEERING MATERIALS

ISSN 2052-5206

\section{Crystal Growth for Beginners. Fundamentals of Nucleation, Crystal Growth and Epitaxy, 3rd edition. Edited by Ivan V Markov. World Scientific, 2017. Pp. 632. Hardback. Price GBP 123.00. ISBN 978-981-3143-42-5}

\author{
S. Roger Qiu*
}

Lawrence Livermore National Laboratory, 7000 East Avenue, Livermore, CA 94550, USA. *Correspondence e-mail: qiu2@|lnl.gov

Crystal growth is a classical discipline lying at the interfaces of Physics, Chemistry, Materials Science and Medicine. The research field has enjoyed an increasing number of young investigators over the past couple of decades, largely due to the burgeoning fields of nanoscience and nanotechnology which aim to fabricate nano-devices and/or to deliver nano-medicines in a process that mimics the exquisite wisdom of biologically controlled crystals. The interdisciplinary nature of these fields has posed a request for introductory monographs on the fundamental concepts of crystallization that are suitable for the diverse scientific backgrounds of young researchers. Among many of the introductory crystal growth books, the Crystal Growth for Beginners (3rd edition) by Ivan Markov is an excellent one that can serve the purpose and is worth reading.

The book is well organized and is pedagogical. By discussing crystallization in pure systems, the author introduces and describes the important concepts, physical parameters and theoretical models pertaining to nucleation and growth of crystals. The book contains five chapters over 600 pages. The chapters are 1. Crystal-Ambient Phase Equilibrium, 2. Nucleation, 3. Crystal Growth, 4. Epitaxial Growth and 5. The Shoulder on Which we Stand. The current edition builds on the author's previous edition of the book with the exact same book title (Markov, 2003). The first four chapters are similar with the current version including some minor additions of topics and figures. Chapter 5 is completely new.

In the first chapter, the concept of crystal formation from phase equilibrium is introduced and the importance of chemical potential in crystallization is addressed. Through the microscopic view of one-component systems, the fundamental concepts, kinetics and thermodynamics of kinks, steps, flat surfaces, rough surfaces and equilibrium crystal shapes are discussed. In addition, the derivation of the impactful pure thermodynamic Thomson-Gibbs equation (p. 12) by Stranski and Kaischew utilizing a kinetic approach is elegantly presented (p. 45).

The thermodynamics and kinetics of nucleation via a classical pathway are addressed in the second chapter. The organization of the chapter is excellent, which makes it easy for beginners to grasp the important underlying physics involved in nucleation. For thermodynamics, homogenous and heterogenous nucleation of three-dimensional (3D) nuclei are discussed, followed by the presentation of nucleation of two-dimensional (2D) nuclei on a foreign substrate. For kinetics, the rate of nucleation is fully described. In this section, the author started the topic by first showing the general expression of the steady state rate of nucleation through a well-designed derivation with the assumptions of the model clearly delineated (pp. 105-107). Based on this expression, the rates of nucleation from homogeneous, heterogeneous, $3 \mathrm{D}$ and $2 \mathrm{D}$ in solutions and melts are developed. Furthermore, the atomistic theory of heterogeneous nucleation (pp. 129-143) is also described in detail. Readers with an interest in thin film deposition may find this section useful. This chapter is closed with a description of Ostwald's step rule.

The third chapter is devoted to the concept of crystal growth. In the first half of the chapter, important physical parameters involved in crystal growth, such as growth rate, kinetic coefficient, critical length and their relationships to supersaturation are presented. They are described and discussed in great deal through the growth from different sources, 
i.e. growth from rough surfaces as well as flat surfaces via either 2D nucleation or screw dislocations (including the Frank-Reed type). A new illustration (p. 237) is added in this edition to demonstrate better the three modes of growth through 2D nucleation, namely the mononuclear, polynuclear layer-by-layer growth and the multilayer growth. The models are derived for different systems including melts, solutions and vapors. The second part of the chapter discusses some examples of crystal growth systems and demonstrates how the models are applied to these specific cases. Section 3.3.1, new to this 3rd edition, describes the growth of nanowires by a vaporliquid-solid mechanism. The author did a nice job in integrating the mechanism in the framework of the classic crystal growth theory including the 'modified' Thomson-Gibbs principle (p. 267). Many recent references are included in the review. The impact of step interaction including step bunching on the growth of a crystal and the kinetic theory of crystal growth is also presented.

In the chapter Epitaxial Growth, the cases of homoepitaxy and heteroepitaxy are discussed. Several models of epitaxial growth including those from Volmer-Web and StranskiKrastanov are explored and the models by Frank and van der Merwe are described in detail (Section 4.2.4). Through the review of results obtained mostly from semiconductor materials, the mechanisms of growth in epitaxy films are fully presented. The authors also added two new figures (pp. 478479) and a general review to demonstrate the idea of 'the minimum-energy pathway' in the mechanism of $2 \mathrm{D}$ to $3 \mathrm{D}$ transformation in thin epitaxial films. The effect of surfactant on the thermodynamics and kinetics of epitaxy growth is discussed at the end of the chapter. Researchers working on crystal growth of semiconductor thin films may find this chapter useful.

The final chapter is newly added and provides a vivid biographical description of the early pioneers (including
Cabrera, Frank, Gibbs and van der Merwe, to name but a few) who have set the foundation for the science and technology of modern crystal growth. It is refreshing to read the monographs for each founder as the description of the life story is hard to come by, and for newcomers in the crystal growth field, the inside stories may serve as stimuli to help them easily absorb those seemly 'dry' analytical equations and feel the theoretical models more tangible.

.As Hurle had commented, this is a book of crystal growth of 'Pure Materials' and that the effects of dopants on crystal growth is largely absent (Hurle, 2004). The inclusion of topics on the effect of an impurity (or dopant) on the nucleation and growth of a crystal at an elementary level would make a natural addition to the book for completion. For beginner readers from fields of industrial crystals, energy-harnessing materials, pharmaceutics and bio-inspired materials synthesis this would be great benefit.

The title Crystal Growth for Beginners is slightly misleading. In fact, this book is more suitable for advanced beginners. If you are a young investigator or a graduate student whose research involves in understanding the fundamentals of crystallization including nucleation and growth, this book will be a treat for you. Readers who have a strong background in physical chemistry or thermal physics may find the book easy to read. Nevertheless, this book should be a good reference to have on the bookshelf if you are an experienced researcher whose interest crosses the path with the general topics of crystal growth.

\section{References}

Hurle, D. T. J. (2004). J. Appl. Cryst. 37, 352-353.

Markov, I. V. (2003). Crystal Growth for Beginners. 2nd ed. New Jersey: World Scientific Press. 